\title{
The Galactic distribution of Wolf-Rayet stars: an optical and infrared approach
}

\author{
Karel A. van der Hucht \\ SRON Nationaal Instituut voor Ruimte Onderzoek, \\ Sorbonnelaan 2, NL-3584 CA Utrecht, Nederland
}

\begin{abstract}
The recent VIIth Catalogue of Galactic Wolf-Rayet Stars (van der Hucht 2001), lists 227 Population I WR stars, comprising 127 WN, 87 WC, 10 $\mathrm{WN} / \mathrm{WC}$ and 3 WO stars. Additional discoveries since then bring the census to $\gtrsim 253 \mathrm{WR}$ stars, including $\gtrsim 27 \mathrm{WNL}$ and $13 \mathrm{WCL}$ stars within $50 \mathrm{pc}$ of the Galactic Center. A re-determination of the optical photometric distances and the galactic distribution of WR stars shows in the solar neighbourhood $(d<3 \mathrm{kpc})$ a projected surface density of $2.7 \mathrm{WR}$ stars per $\mathrm{kpc}^{2}$, a $N_{\mathrm{WC}} / N_{\mathrm{WN}}$ number ratio of 1.3, and a WR binary frequency of $40 \%$. Compared with other Local Group galaxies, the $N_{\mathrm{WC}} / N_{\mathrm{WN}}$ number ratio in the solar neighborhood is a factor $\sim 2$ above the observed metallicity-dependent trend. This could imply that some 30 galactic WN stars in the $d<3 \mathrm{kpc}$ volume are still hiding. Preliminary results of infrared photometric distance determinations are shown. The galactocentric distance $\left(R_{\mathrm{WR}}\right)$ distribution per subtype shows $\overline{R_{\mathrm{WN}}}$ and $\overline{R_{\mathrm{WC}}}$ decreasing with later WN and WC subtypes. The observed trend is more indicative of WNE $\rightarrow$ WCE and WNL $\rightarrow$ WCL subtype evolution than of WNL $\rightarrow$ WNE and WCL $\rightarrow$ WCE subtype evolution.
\end{abstract}

\section{Introduction}

WR stars are characterized by strong $\mathrm{He}, \mathrm{N}, \mathrm{C}$ and $\mathrm{O}$ emission lines, originating in their hot stellar winds with terminal velocities in the range $v_{\infty} \simeq 400$ $5000 \mathrm{~km} \mathrm{~s}^{-1}$, which drive mass loss rates of the order of $\dot{M}=10^{-5} \mathrm{M}_{\odot} \mathrm{yr}^{-1}$. In these WR winds also their free-free $\mu \mathrm{m}$-to-cm continuum emission originates, which allows mass loss rate determinations, given estimates of wind abundances, ionization, clumping rates, terminal wind velocities and distances of the WR stars (Wright \& Barlow 1975; Crowther 1999). Recent studies of coeval open clusters and $\mathrm{OB}$ associations suggest that the minimum initial stellar mass for reaching the WR phase is $18 \mathrm{M}_{\odot}$ in the Milky Way (Massey et al. 2001), $30 \mathrm{M}_{\odot}$ in the LMC and $70 \mathrm{M}_{\odot}$ in the SMC (Massey et al. 2000). Observed Galactic WR masses in binary systems fall in the range 2.3-55 $\mathrm{M}_{\odot}$ (van der Hucht 2001).

Reviews of the WR phenomenon have been presented by, e.g., van der Hucht (1992), Maeder \& Conti (1994), and in Proc. IAU Symp. No. 143 (van der Hucht \& Hidayat 1991), in Proc. IAU Symp. No. 163 (van der Hucht \& Williams 1995), in Proc. $33^{\text {rd }}$ Liège Intern. Astroph. Coll. (Vreux et al. 1996), and in Proc. IAU Symp. No. 193 (van der Hucht, Koenigsberger \& Eenens 1999).

The VIIth Catalogue of Galactic Wolf-Rayet Stars (van der Hucht 2001, follow-up of the VIth Catalogue of van der Hucht et al. 1981), brings the number 

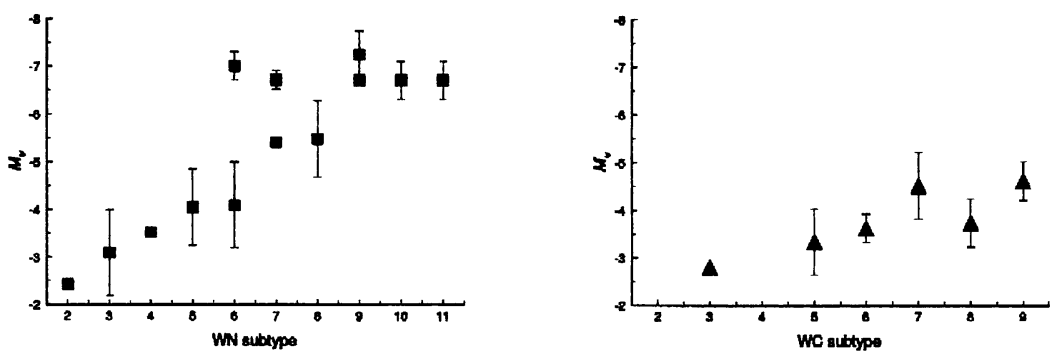

Figure 1. $\overline{M_{v}(\mathrm{WR})} v s$. WR subtype. Left: the upper values at WN6, WN7 and WN9 refer to, respectively, WN6ha WN7ha and WN9ha stars. Right: the point at WC3 refers to WO stars only. Error bars denote the standard deviations of the averages. From van der Hucht (2001).

of known Galactic Population I WR stars to 227, derives photometric distances and shows their Galactic distribution. Since then, 11 new WR stars (6 WN and $5 \mathrm{WC}, V>17 \mathrm{mag}$ ) have been discovered optically in the open cluster Westerlund $1(d \approx 5 \mathrm{kpc})$ by Negueruela \& Clark (these Proceedings, see also Clark \& Negueruela 2002); and one new WC8 star $(B=18.1)$ has been discovered in the near-IR by Pasquali et al. (2002) in the neighborhood of Cyg OB2 and Cyg OB9 (at $d \simeq 1.8 \mathrm{kpc}$ ).

In addition, seven new WNL stars have been discovered in the near-IR in the Galactic Center Cluster by Genzel et al. (2000) and Paumard et al. (2001); two new WC stars have been discovered in and near the Quintuplet Cluster by Homeier et al. (2003, and in these Proceedings); and $\geq 5$ new WNL stars have been identified by in the Arches Cluster by Figer et al. (2002). This brings the known WR population near the Galactic Center to $\gtrsim 40$.

The Galactic WR census is still rather incomplete, due to the ubiquitous interstellar extinction in the plane of the Milky Way. Especially at near-IR and IR wavelengths, numerous WR stars are waiting to be discovered (see also Diehl, these Proceedings).

It is important to discover and monitor WR stars, while each of them is a unique physics laboratory, a tracer of star formation in spiral arms, and a representative of the one but final phase in the evolution of massive stars, probably followed by a Type Ib/Ic supernova explosion (e.g., Maeder \& Conti 1994; Langer \& Woosley 1996; Kaper \& Cherepashchuk 2001; Chu 2002; Cherepashchuk in these Proceedings), either single in origin or binary (Langer \& Heger 1999; Wellstein \& Langer 1999). One cannot hope to understand, e.g., the formation of black holes without understanding the late stages of massive star evolution. And where statistically the next Galactic supernova is overdue for already more than a century, it is of obvious relevance to gather detailed knowledge about each of its potential progenitors.

\section{Distances of Galactic Wolf-Rayet stars}

To derive heliocentric photometric distances of WR stars, van der Hucht (2001) re-investigated the absolute visual magnitudes $M_{v}$ and intrinsic colours $(b-v)$ 。 


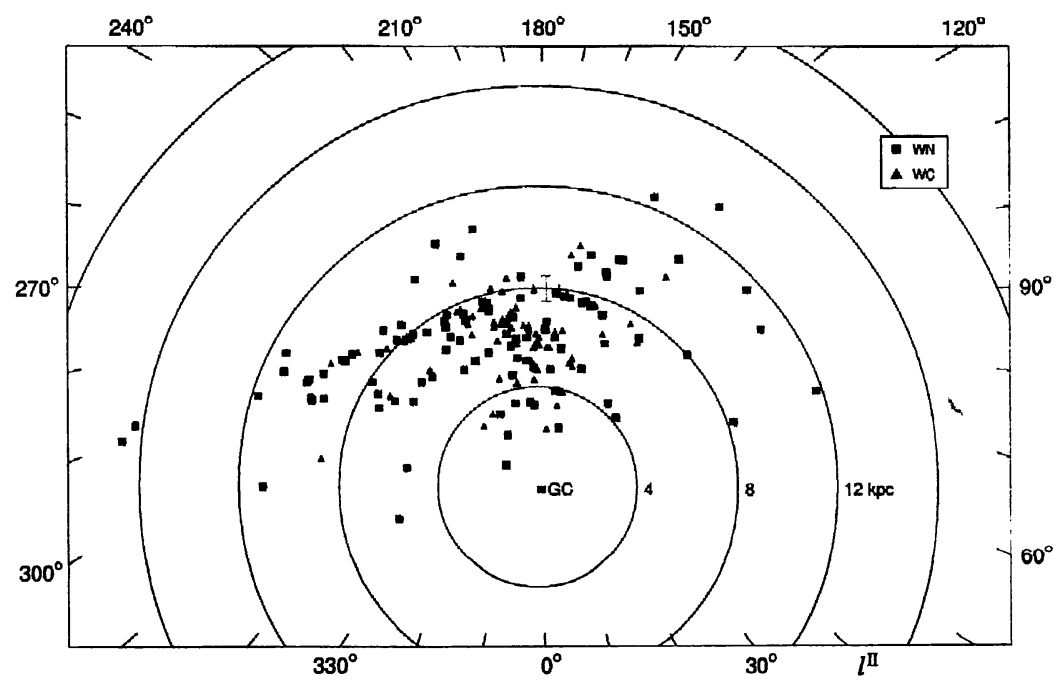

Figure 2. The Galactic Wolf-Rayet star distribution $\left(l^{\mathrm{II}}, d\right)$ projected on the Galactic plane for the stars in the VIth Catalogue. The Sun is indicated by + . The distance from the Sun to the Galactic Center is adopted at $8.0 \mathrm{kpc}$ (Reid 1993). The point at the Galactic Center harbours $\gtrsim 50$ WR stars. From van der Hucht (2001).

of WR stars in open clusters and OB associations, in the filter system of Smith (1968a), correcting for contributions from binary companions where warranted. The resulting average $M_{v}$-values are plotted in Figure 1 as a function of WR sub-type.

Subsequently, photometric distances of fields WR stars followed directly from their apparent $b v$ magnitudes and subtype-dependent intrinsic parameters $M_{v}$ and $(b-v)_{\circ}$, again corrected for companion contributions in case of binarity. The errors in the resulting photometric distances of WR field stars correspond at least to the range of $M_{v}$-values for the particular subtype (see Figure 1). We estimate the average error in the WR field star distances as $\sim 50 \%$.

The distance of the Galactic Center was adopted at $d_{\mathrm{GC}}=8.0 \pm 0.5 \mathrm{kpc}$ (Reid 1993; McNamara et al. 2000). For the 26 WR objects in the VIIth Catalogue within $50 \mathrm{pc}$ of the Galactic Center (15 WNL and 11 WCL stars) that distance was adopted, as well as $A_{V}=29 \pm 5 \mathrm{mag}$ (Figer et al. 1999a), i.e., $A_{v}=32 \mathrm{mag}$.

\section{Wolf-Rayet star longitude distribution}

\subsection{Galactic structure}

The WR Galactic distribution $\left(l^{\mathrm{II}}, d\right)$ is plotted in Figure 2, which reflects a Galactic spiral structure (compare with the Galactic distribution of $\mathrm{H}$ II regions by Georgelin \& Georgelin 1976, fig. 11; up-dated by Russeil 1998, fig. 8-4; Russeil 2003, fig. 4; and Russeil in these Proceedings). 
Table 1. Numbers, number ratios, and surface densities $\sigma_{\mathrm{WR}}$ of the 77 WR stars in the VIIth Catalogue with $d<3 \mathrm{kpc}$ as a function of galactocentric distance $R$ (adopting $R_{\odot}=8.0 \mathrm{kpc}$ ).

\begin{tabular}{|c|c|c|c|c|c|c|c|c|c|c|}
\hline $\begin{array}{c}R \\
(\mathrm{kpc})\end{array}$ & $N_{\mathrm{WR}}$ & $N_{\mathrm{WN}}$ & $N_{\text {WC }}$ & $N_{\text {wo }}$ & $\frac{N_{\mathrm{WC}}}{N_{\mathrm{WN}}}$ & $N_{\mathrm{WN}}^{\text {binary }}$ & $N_{\text {WC }}^{\text {binary }}$ & $N_{\mathrm{WR}}^{\text {binary }}$ & $\begin{array}{r}\text { area } \\
\left(\mathrm{kpc}^{2}\right)\end{array}$ & $\begin{array}{c}\sigma_{\mathrm{WR}} \\
\left(\mathrm{kpc}^{-2}\right)\end{array}$ \\
\hline $\begin{array}{c}5-7 \\
7-9 \\
9-11\end{array}$ & $\begin{array}{r}39 \\
31 \\
7\end{array}$ & $\begin{array}{r}14 \\
16 \\
4\end{array}$ & $\begin{array}{r}25 \\
14 \\
3\end{array}$ & 1 & $\begin{array}{l}1.8 \\
0.9 \\
0.8\end{array}$ & $\begin{array}{l}3 \\
5 \\
2\end{array}$ & $\begin{array}{c}10 \\
10 \\
1\end{array}$ & $\begin{array}{l}33 \% \\
48 \% \\
43 \%\end{array}$ & $\begin{array}{r}9.17 \\
11.83 \\
7.27\end{array}$ & $\begin{array}{l}4.3 \\
2.6 \\
1.0\end{array}$ \\
\hline total & 77 & 34 & 42 & 1 & 1.3 & $\begin{array}{c}10 \\
(29 \%)\end{array}$ & $\begin{array}{c}21 \\
(50 \%)\end{array}$ & $40 \%$ & 28.27 & 2.7 \\
\hline
\end{tabular}

As stated earlier by Conti \& Vacca (1990), the overall similarity of Figure 2 and previous diagrams for WR stars by Smith (1968b, fig. 1), van der Hucht et al. (1988, fig. 3) and Conti \& Vacca (1990, fig. 1) demonstrates that the photometric distance determination procedure is fairly 'robust', on average. However, distances for individual WR stars can differ substantially. We confirm earlier conclusions by Roberts (1962), Smith (1968b), Conti et al. (1983), van der Hucht et al. (1988) and Conti \& Vacca (1990), that the galactocentric distance distribution of WR stars is subtype-dependent, notably with the WCL stars confined towards the Galactic Center, and that the Galactic anti-center quadrant (Orion spur) is devoid of WR stars.

In the volume with $d<3 \mathrm{kpc}$ the VIIth Catalogue lists $77 \mathrm{WR}$ stars (16 more than the census of van der Hucht et al. 1988) with $N_{\mathrm{WN}}: N_{\mathrm{WC}}: N_{\mathrm{WO}}=34: 42: 1$. Since the WN and WC phases are consecutive in the evolution of massive stars, this implies that in the solar neighbourhood the average WC-phase life-time is $\sim 1.3$ times longer than the average WN-phase life-time. When divided in shells around the Galactic Center, we count in the $d<3 \mathrm{kpc}$ volume a number ratio $N_{\mathrm{WC}} / N_{\mathrm{WN}}=0.9$ for $7<R_{\mathrm{WR}}<9 \mathrm{kpc}$, while $N_{\mathrm{WC}} / N_{\mathrm{WN}}=1.8$ for $5<R_{\mathrm{WR}}<7$ kpc. Projected on the Galactic plane the WR surface density in that volume is $\sigma_{\mathrm{WR}}=2.7 \mathrm{kpc}^{-2}$.

The overall subtype distribution and binary frequency breakdown in the volume with $d<3 \mathrm{kpc}$ is listed in Table 1 . The observed WR binary frequency in that volume ( $31 \mathrm{WR}$ binaries) is $40 \%$. The WC binary frequency in that volume $(50 \%)$ is almost twice the WN binary frequency $(29 \%)$.

The $N_{\mathrm{WC}} / N_{\mathrm{WN}}$ ratio in the Milky Way within $3 \mathrm{kpc}$ from the Sun found here, is a factor of 2.5 above the trend found in Local Group galaxies by Massey \& Johnson (1998, see also Massey in these Proceedings, figure 2), who concluded that the WN stars appear underrepresented in this volume. That would imply that within $d<3 \mathrm{kpc}$ some 30 Galactic WN stars are still hiding.

\subsection{Wolf-Rayet subtype distribution and evolution}

The VIIth Catalogue allows to investigate the WR galactocentric distances $R_{\mathrm{WR}}(\mathrm{kpc})=\sqrt{d^{2}+64+16 d \cos b^{\mathrm{II}} \cos l^{\mathrm{II}}}$. Figure 3 shows the WR galactocen- 


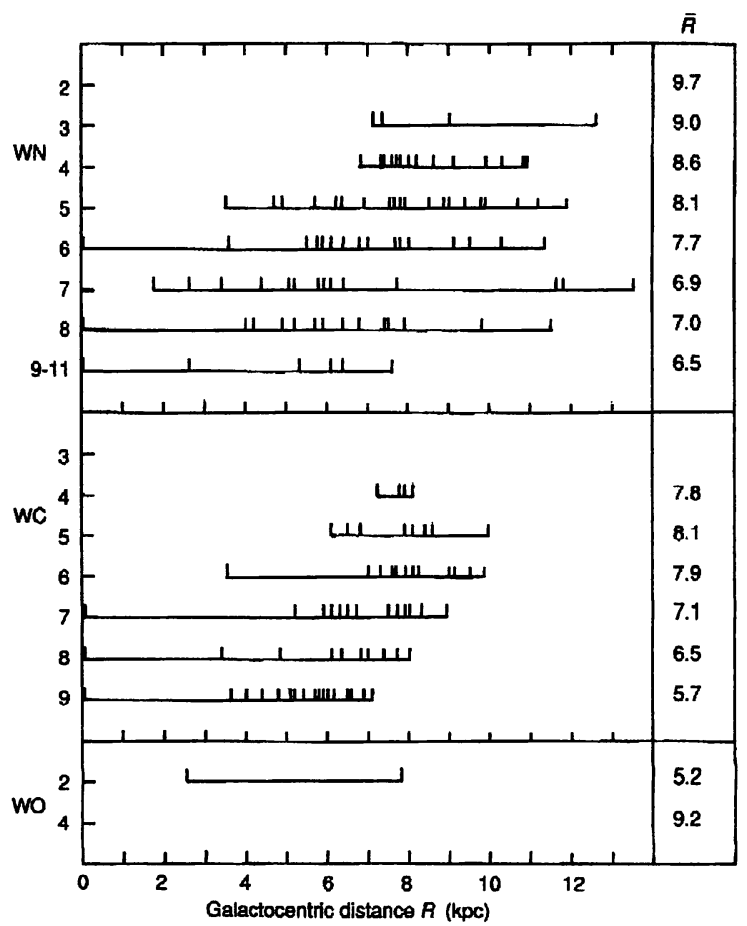

Figure 3. The galactocentric Wolf-Rayet subtype distribution. Right: the average $R_{\text {WR }}$ per subtype, excluding the WR stars at the Galactic Center. $R_{\odot}=8.0 \mathrm{kpc}$ adopted. From van der Hucht (2001).

tric distribution per subtype. As quantified in the right side column of Figure 3, the WN stars show a remarkable trend for $\overline{R_{\mathrm{WN}}}$ values per subtype to decrease with later WN subtype. The WC stars show a similar trend for $\overline{R_{\mathrm{WC}}}$ values per WC subtype to decrease with later WC subtype up to WC6. For WC6-4 stars

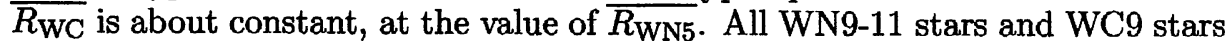
are located within the solar circle $\left(R_{\odot}=8 \mathrm{kpc}\right)$. We do not observe WC stars beyond $R=10 \mathrm{kpc}$, but WN3-8 stars are observed there.

Figure 3 poses no strict constraints on WR subtype evolution, but shows that evolution from the WN2-3 phase to the WC9 phase is unlikely. Figure 3 does not encourage WNL $\rightarrow$ WNE or WCL $\rightarrow$ WCE subtype evolution (favoured by Moffat 1995). The observed similar $R_{\mathrm{WN}}$ and $R_{\mathrm{WC}}$ trends do allow WNE $\rightarrow$ WCE and WNL $\rightarrow$ WCL subtype evolution.

\section{Infrared photometric distances}

While van der Hucht (2001) performed an optical photometric distance determination, van der Hucht et al. (2003) introduce an infrared photometric distance determination, based on $J H K L^{\prime} M$ photometry of 145 Galactic WR stars. This method should yield smaller de-reddening errors, because of reduced reddening compared to the optical wavelength range. Figure 4 shows preliminary results, 

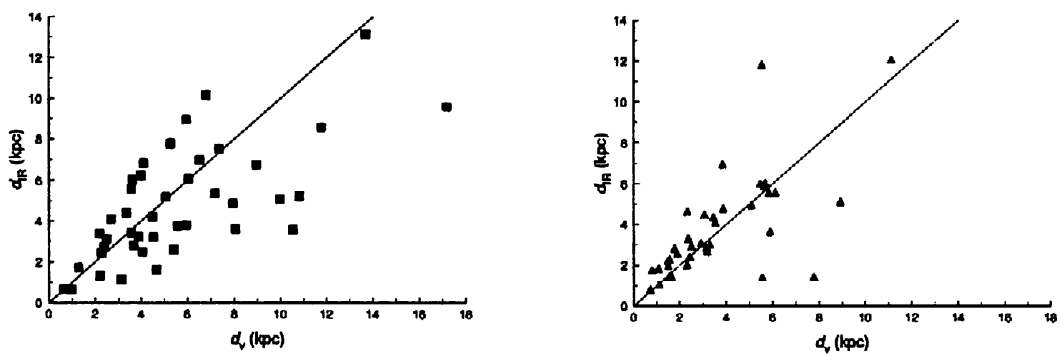

Figure 4. Infrared vs. optical photometric distances for Wolf-Rayet field stars. Left: $d_{v}$ vs. $d_{\mathrm{IR}}$ for WN stars ( $\left.\mathbf{\square}\right)$. Right: $d_{v} v s$. $d_{\mathrm{IR}}$ for WC stars $(\mathbf{\Delta})$.

comparing optical and infrared photometric distances of WR field stars. Details will be given by van der Hucht et al. (2003).

\section{Wolf-Rayet stars in the Galactic Center}

The Galactic Center region proves to be an area very rich in WR stars. The VIIth Catalogue lists within $50 \mathrm{pc}$ from the Galactic Center 15 WNL and 11 WCL stars, at near-IR wavelengths discovered by Blum et al. (1995); by Krabbe et al. (1995) in the Galactic Center Cluster; by Figer et al. (1995, 1999a, 1999b) in the Quintuplet Cluster (AFGL 2004); and by Cotera et al. (1999) in the Arches Cluster, although some of them are questioned by Paumard et al. (2001). Earlier classifications of so-called He I stars have been revised by Crowther (private communication) in the VIIth Catalogue into WN9-11 stars.

In the meantime, the number of WR stars discovered in the Galactic Center Cluster has increased with seven additional WNL stars (Genzel et al. 2000: 13S SE, 16CC and MPE-8.3,-5.7; Paumard et al. 2001: ID 180, IRS 7E2, HeI N3 and HeI N2); that in and near the Quintuplet Cluster with two new WC stars (Homeier et al. 2003, and in these Proceedings); and that in the Arches Cluster with $\gtrsim 5$ new WNL stars (Figer et al. 2002). Moreover, in the Quintuplet Cluster at least five potential WCL stars have been identified, of which the heated circumstellar dust radiates sufficiently strongly in the near-IR to masks the WC IR emission-line spectra, thus preventing spectral classification. Moneti et al. (1992, 1994, 2001) coined them cocoon stars, while Figer et al. (1996, 1999a) listed them as 'DWCL?' stars.

Figure 5 suggests a surface density of the Galactic Center WR stars of $\sigma_{\mathrm{WR}} \approx 4200 \mathrm{kpc}^{-2}$ in the $R<50 \mathrm{pc}$ volume.

\section{The total number of Wolf-Rayet stars in the Milky Way}

Since we observe roughly only one quadrant of the Milky Way as projected on the Galactic plane, we can assume that the minimum total number of Galactic WR stars is four times the number of WR stars in the VIIth Catalogue, i.e., $N_{\min }^{\mathrm{WR}} \simeq 1000$.

Various attempts have been made to estimate the total number of WR stars in the Milky Way. E.g., Prantzos \& Cassé (1986), by scaling an earlier observed 


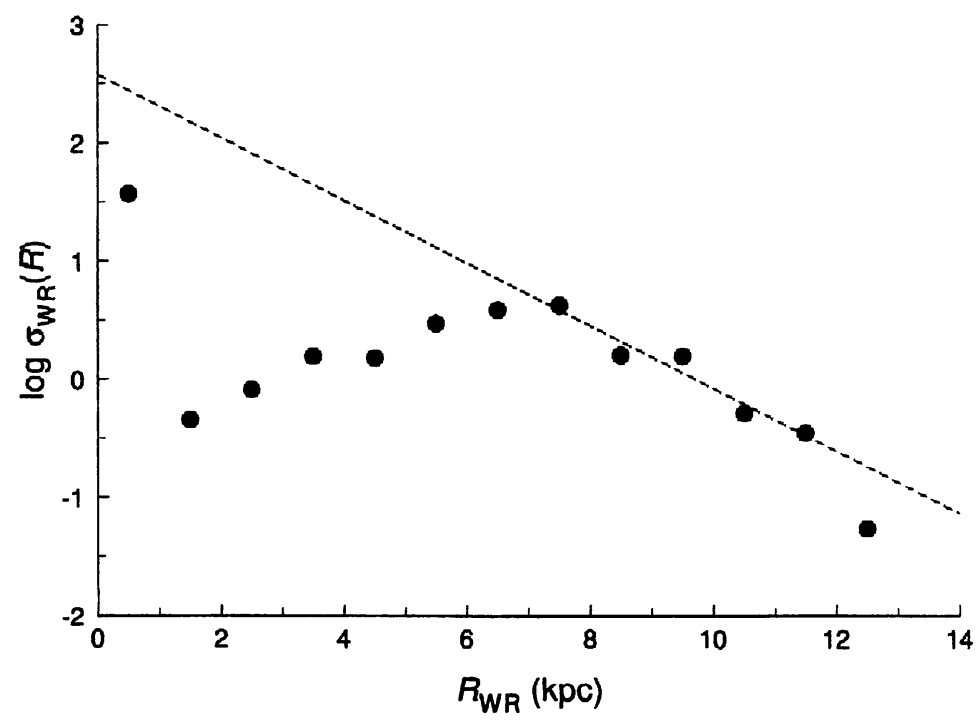

Figure 5. The Galactic WR surface density distribution $\sigma_{\mathrm{WR}}(R)$ vs. galactocentric distance $R_{\mathrm{WR}}$ for the stars in the VIIth Catalogue. The dashed line, fitting the five data points with $R_{\mathrm{WR}}=7-12 \mathrm{kpc}$, corresponds to $\log \sigma_{\mathrm{WR}}(R)=-0.26 R_{\mathrm{WR}}+2.58$.

WR density distribution (Hidayat et al. 1982) to that of molecular hydrogen (Sanders et al. 1984) and adopting that the number ratio of WR stars to O-type stars is metallicity-dependent as $N_{\mathrm{WR}} / N_{\mathrm{O}} \propto Z^{1.7}$ (Maeder 1984), estimated that $N_{\text {total }}^{\mathrm{WR}} \simeq 8000$. The present total number of O-B2 stars in the Milky Way has been estimated at $\sim 60000$ (Reed 2000).

Van der Hucht (2001) estimates the present total number of WR stars in the Milky Way, on the basis of the observed Galactic WR surface density distribution $\sigma_{\mathrm{WR}}(R)$ only. Assuming that the WR inventory in the shell $R=7-12 \mathrm{kpc}$ is complete, the data in Figure 5 imply that $\log \sigma_{\mathrm{WR}}(R)=-0.26 R+2.58$, and thus that $N_{\text {total }}^{\mathrm{WR}} \simeq 6500$, with $\sim 3600 \mathrm{WR}$ stars within $3 \mathrm{kpc}$ from the Galactic Center (where to date $\gtrsim 40 \mathrm{WR}$ stars have been discovered).

Adopting as the average WR star life-time $t_{\mathrm{WR}} \simeq 4 \times 10^{5} \mathrm{yr}$ (Maeder \& Meynet 1994), we can expect for the average time-interval $\tau_{\mathrm{WR}}$ between two consecutive Galactic WR deaths (possibly as supernovae) that $\tau=t_{\mathrm{WR}} / N_{\mathrm{total}}^{\mathrm{WR}} \simeq 60 \mathrm{yr}$, or only in the observable quadrant of the Milky Way, $\tau \simeq 250 \mathrm{yr}$.

\section{Summary and conclusions}

The VIIth Catalogue of Galactic Wolf-Rayet Stars lists 227 Population I WR stars, comprising $127 \mathrm{WN}$ stars, $87 \mathrm{WC}$ stars, $10 \mathrm{WN} / \mathrm{WC}$ stars and $3 \mathrm{WO}$ stars. Additional recent new discoveries bring the total number to $\gtrsim 240$ known Galactic WR stars, including $\gtrsim 27$ WNL and 13 WCL stars within $50 \mathrm{pc}$ of the Galactic Center. 
A re-determination of optical photometric distances and the Galactic distribution of the WR stars in the VIIth Catalogue shows in the volume $d<3 \mathrm{kpc}$ a projected surface density $\sigma_{\mathrm{WR}}=2.7 \mathrm{kpc}^{-2}$, a number ratio $N_{\mathrm{WC}} / N_{\mathrm{WN}}=1.3$, and a WR binary frequency of $40 \%$. The solar neighborhood number ratio of $N_{\mathrm{WC}} / N_{\mathrm{WN}}=1.3$ is a factor of $\sim 2$ above the metallicity-dependent trend seen in other Local Group galaxies. This could imply that at least 30 Galactic WN stars in the $d<3 \mathrm{kpc}$ volume are still hiding. shown.

Preliminary results of infrared photometric distance determinations are

The galactocentric distance $\left(R_{\mathrm{WR}}\right)$ distribution per subtype shows $\overline{R_{\mathrm{WR}}}$ decreasing with later WR subtype for both the WN and WC sequences. The observed trend is more indicative of WNE $\rightarrow$ WCE and WNL $\rightarrow$ WCL subtype evolution, rather than of WNL $\rightarrow \mathrm{WNE}$ and $\mathrm{WCL} \rightarrow \mathrm{WCE}$ subtype evolution.

\section{References}

Blum, R.D., Sellgren, K., DePoy, D.L. 1995, ApJ (Letters) 440, L17

Chu, Y.-H. 2002, in: A.F.J. Moffat \& N. St-Louis (eds.), Interacting Winds from Massive Stars, ASP-CS 260, 109

Clark, J.S., Negueruela, I. 2002, A\&A (Letters) 396, L25

Conti, P.S., Garmany, C.D., de Loore, C., Vanbeveren, D. 1983, ApJ 274, 302

Conti, P.S., Vacca, W.D. 1990, AJ 100, 431

Cotera, A.S., Simpson, J.P., Erickson, E.F., Colgan, S.W.J., Burton, M.G., Allen, D.A. 1999, ApJ 510, 747

Crowther, P.A. 1999, in: K.A. van der Hucht, G. Koenigsberger \& P.R.J. Eenens (eds.), Wolf-Rayet Phenomena in Massive Stars and Starburst Galaxies, Proc. IAU Symp. No. 193 (San Francisco: ASP), p. 116

Figer, D.F., McLean, I.S., Morris, M. 1995, ApJ (Letters) 447, L29

Figer, D.F., Morris, M., McLean, I.S. 1996, in: R. Gredel (ed.), The Galactic Center, ASP-CS 102, 263.

Figer, D.F., McLean, I.S., Morris, M. 1999a, ApJ 514, 202

Figer, D.F., Morris, M., Geballe, T.R., Rich, R.M., Serabyn, E., McLean, I.S., Puetter, R.C., Yahil, A. 1999b, ApJ 525, 759

Figer, D.F., Najarro, F., Gilmore, D., Morris, M., Kim, S.S., Serabyn, E., McLean, I.S., Gilbert, A.M., Graham, J.R., Larkin, J.E., Levenson, N.A., Teplitz, H.I. 2002, ApJ 581, 258

Genzel, R., Pichon, C., Eckart, A., Gerhard, O.E., Ott, T. 2000, MNRAS 317, 348

Georgelin, Y.M., Georgelin, Y.P. 1976, A\&A 49, 57

Hidayat, B., Supelli, K., van der Hucht, K.A. 1982, in: C. de Loóre \& A.J. Willis (eds.), Wolf-Rayet Stars: Observations, Physics, Evolution, Proc. IAU Symp. No. 99 (Dordrecht: Reidel), p. 27

Homeier, N.L., Blum, R.D., Conti, P.S., Damineli, A. 2003, A\&A 397, 585

van der Hucht, K.A., Conti, P.S., Lundström, I., Stenholm, B. 1981, Space Sci. Reviews 28, 227 [VIth Catalogue]

van der Hucht, K.A., Hidayat, B., Admiranto, A.G., Supelli, K.R., Doom, C. 1988, A\&A 199, 217

van der Hucht, K.A., Hidayat, B. (eds.) 1991, Wolf-Rayet Stars and Interrelations with Other Massive Stars in Galaxies, Proc. IAU Symp. No. 143 (Dordrecht: Kluwer)

van der Hucht, K.A. 1992, The A\&A Review 4, 123 
van der Hucht, K.A., Williams, P.M. (eds.) 1995, Wolf-Rayet Stars: Binaries, Colliding Winds, Evolution, Proc. IAU Symp. No. 163 (Dordrecht: Kluwer)

van der Hucht, K.A., Koenigsberger, G., Eenens, P.J.R. (eds.) 1999, Wolf-Rayet Phenomena in Massive Stars and Starbursts Galaxies, Proc. IAU Symp. No. 193 (San Francisco: ASP)

van der Hucht, K.A. 2001, New Astron. Reviews 45, 135 [VIIth Catalogue]

van der Hucht, K.A., Williams, P.M., Setia Gunawan, D.Y.A. 2003, in preparation

Kaper, L., Cherepashchuk, A. 2001, in: L. Kaper, E.P.J. van den Heuvel \& P.A. Woudt (eds.), Black Holes in Binaries and Galactic Nuclei, Proc. ESO Astrophys. Symp. (Berlin: Springer), p. 277

Krabbe, A., Genzel, R., Eckart, A., Najarro, F., Lutz, D., Cameron, M., Kroker, H., Tacconi-Garman, L.E., Thatte, N., Weitzel, L., Drapatz, S., Geballe, T., Sternberg, A., Kudritzki, R.-P. 1995, ApJ (Letters) 447, L95

Langer, N., Woosley, S.E. 1996, in: C. Leitherer, U. Fritze-von Alvensleben \& J. Huchra (eds.), From Stars to Galaxies. The Impact of Stellar Physics on Galaxy Evolution, ASP-CS 98, 220

Langer, N., Heger, A. 1999, in: K.A. van der Hucht, G. Koenigsberger \& P.R.J. Eenens (eds.), Wolf-Rayet Phenomena in Massive Stars and Starburst Galaxies, Proc. IAU Symp. No. 193 (San Francisco: ASP), p. 187

Maeder, A. 1984, Adv. Space Res. 4, 55

Maeder, A., Conti, P.S. 1994, Ann. Review Astron. Astrophys. 32, 227

Maeder, A., Meynet, G. 1994, A\&A 287, 803

Massey, P., Johnson, O. 1998, ApJ 505, 793

Massey, P., Waterhouse, E., DeGioia-Eastwood, K. 2000, AJ 119, 2214

Massey, P., DeGioia-Eastwood, K., Waterhouse, E. 2001, AJ 121, 1050

McNamara, D.H., Madsen, J.B., Barnes, J., Ericksen, B.F. 2000, PASP 112, 202

Moffat, A.F.J. 1995, in: K.A. van der Hucht \& P.M. Williams (eds.), Wolf-Rayet Stars: Binaries, Colliding Winds, Evolution, Proc. IAU Symp. No. 163 (Dordrecht: Kluwer), p. 213

Moneti, A., Glass, I.S., Moorwood, A.F.M. 1992, MNRAS 258, 705

Moneti, A., Glass, I.S., Moorwood, A.F.M. 1994, MNRAS 268, 194

Moneti, A., Stolovy, S., Blommaert, J.A.D.L., Figer, D.F., Najarro, F. 2001, A\&A 366, 106

Pasquali, A., Comerón, F., Gredel, R., Torra, J., Figueras, F. 2002, A\&A 396, 533

Paumard, T., Maillard, J.P., Morris, M., Rigaut, F. 2001, A\&A 366, 466

Prantzos, N., Cassé, M. 1986, ApJ 307, 324

Reed, B.C. 2000, AJ 120, 314

Reid, P. 1993, Ann. Review Astron. Astrophys. 31, 345

Roberts, M.S. 1962, AJ 67, 79

Russeil, D. 1998, Étude Multispectrale des Régions d'Hydrogène Ionisé dans Notre Galaxie, thesis Univ. de Provence, Aix-Marseille I., France

Russeil, D. 2003, A\&A 397, 133

Sanders, D.B., Solomon, P.M., Scoville, N.Z. 1984, ApJ 276, 182

Smith, L.F. 1968a, MNRAS 138, 109

Smith, L.F. 1968b, MNRAS 141, 317

Vreux, J.-M., Detal, A., Fraipont-Caro, D., Gosset, E., Rauw, G. (eds.) 1996, WolfRayet Stars in the Framework of Stellar Evolution, Proc. $33^{\text {rd }}$ Liège Int. Astroph. Coll. (Liège: Univ. of Liège) 
Wellstein, S., Langer, N. 1999, A\&A 350, 148

Wright, A.E., Barlow, M.J. 1975, MNRAS 170, 41

\section{Discussion}

MASSEY: I'm wondering if you have considered the expected intrinsic dispersion in absolute magnitude of WRs - if you consider the (large) mass range that becomes an early WN or late WC according to the evolutionary models, wouldn't you expect a large dispersion in $\mathrm{M}_{v}$ ?

VAN DER HUCHT: Indeed, we will be always left with some intrinsic scatter in $\mathrm{M}_{v}$ due to mass differences within the same spectral subtype. But in my opinion, the current large dispersion is for a large fraction due to incertainties of the adopted distances of open clusters and $\mathrm{OB}$ associations.

WALBORN: I think that the scatter in WNL absolute magnitudes is dominated by intrinsic spread rather than errors. In the LMC, one finds a range of -5 to nearly -8 . This in turn likely reflects different formation channels: mass-transfer binaries, post-RSG, and extremely massive stars in giant $\mathrm{H}$ II regions.

VAN DER HUCHT: As said above, there is likely to be intrinsic scatter. But, I wonder whether a scatter of 3 magnitudes perhaps reflects undetected multiplicity.

MAíz-APELLÁNIZ: I could not agree more with your comment on the need for an updated catalogue of O-type stars (as a follow up of that of Garmany et al. 1982). We are currently working on precisely that (see our poster, these Proceedings) and we will soon make it available.

VAN DER HUCHT: Wonderful.

KOENIGSBERGER: Is the ratio WR/O-stars in clusters similar or different from this ratio for the field stars?

VAN DER HUCHT: I think it is different because incompleteness among field stars is even larger than that among cluster stars. But perhaps it should also be different because WR stars are older and could have drifted away from clusters, more than O-type stars.

GIEs: How many of the WR stars in your catalogue might be low mass objects?

WALBORN: Comment: PN central stars in the WR sample would be only [WC].

VAN DER HUCHT: Among the WR stars in our VIIth Catalogue we doubt only one: WR 109 (V617 Sgr), which is a peculiar object (not even a [WR] central star of a PN). All other stars in our cataloge are true massive Population I WR stars, and properly classified as such. We have not listed known Population II [WC] objects, as we did separately in our VIth Catalogue (van der Hucht et al. 1981). [WN] objects are not known to exist, see the comment by Nolan.

ZINNECKER: Are all Galactic WR stars in open clusters and OB association or are there many WR stars in the field?

VAN DER HUCHT: See the VIIth WR catalogue (van der Hucht 2001): of the listed 227 Galactic WR stars, only 53 are in open culsters and OB associations, or believed to be. The other 184 are supposedly field stars. 